\title{
Economía, política y violencia. Así piensan los salvadoreños a fines de 1998
}

\author{
Instituto Universitario de Opinión Pública
}

\begin{abstract}
Resumen
Entre el 12 y el 15 de diciembre de 1998, el IUDOP realizó un sondeo de opinión pública con una muestra de 1,239 adultos en todo el país, con un margen de error de más o menos el cuatro por ciento. La encuesta, diseñada para recoger la opinión de los salvadoreños a finales de 1998 , revela que la población piensa que la delincuencia ha aumentado en el año que termina y que el problema económico sigue estando igual que antes, aunque hacen mayor énfasis en la pobreza y el desempleo. La pesquisa reveló también que los salvadoreños no están muy entusiasmados por la campaña electoral, y que el partido de gobierno mantiene una ventaja notable en las preferencias ciudadanas de cara a las elecciones presidenciales de 1999.
\end{abstract}

\section{Introducción}

Si existe un problema por el cual los salvadoreños parecen estar tradicional e históricamente preocupados, ése es el de la economía. Efectivamente, desde que el IUDOP comenzó a realizar encuestas de opinión pública en 1986 ha aparecido sin falta la preocupación de los ciudadanos por los temas económicos con diversos matices. Pero esta inquietud ciudadana por la economía va más allá de una simple definición de problema compartido. Para los salvadoreños, los problemas económicos del país constituyen la causa primordial del conflicto armado de los ochenta, representan el mayor desafío para el futuro del desarrollo del país y son unos de los principales causantes de otros dilemas sociales como la delincuencia. Hablar de los problemas económicos y del estado de los mismos año tras año puede ser poco novedoso, pero no por ello es menos necesario. La forma en que los salvadoreños ven sus dificultades económicas y las de la nación puede ser muy útil para entender las opiniones y las conductas sociales en otras esferas públicas. Este artículo pretende eso: revisar la opinión pública sobre la economía de manera 
general y advertir cambios en la misma que puedan estar interactuando con otros aspectos de la opinión pública a finales de 1998.

Por otro lado, violencia y política son dos términos que, en décadas anteriores, siempre aparecían acompañados. Es más, era difícil concebir la política salvadoreña sin su respectiva dosis de violencia. También era extraño encontrar casos de violencia sin que detrás de ellos no hubiese una motivación política. Esa asociación ha marcado en buena medida la historia salvadoreña y lo hizo hasta la firma de los acuerdos de paz, hace aproximadamente siete años. Sin embargo, la violencia ya no aparece tan asociada con la política como en el pasado $y$, exceptuando algunos incidentes ocasionales, ahora la violencia no parece tener motivaciones políticas. Con todo, ambos aspectos de la vida nacional - ahora por separado- siguen dominando la opinión pública, siguen preocupando a los salvadoreños y continúan ocupando las agendas informativas de los medios locales e internacionales. En el año que recién termina esto fue especialmente cierto: la violencia y la política ocuparon la mayor parte de las discusiones públicas, dado que el otro gran tema - el de la economíade interés público cedió terreno.

Este pequeño artículo presenta los resultados de la última encuesta de opinión pública realizada en 1998 y que recoge las opiniones de los ciudadanos sobre el curso del año en cuestión. Los tópicos que dominaron al pensamiento de los ciudadanos siguen siendo la economía, la violencia y la política. Los resultados de la consulta ciudadana que se presentan a continuación permiten tener un panorama del ambiente subjetivo social que dominaba la sociedad salvadoreña unos meses antes de las últimas elecciones presidenciales del siglo.

El arlículo en cuestión se divide en varios apartados. A continuación se presentan los detalles metodológicos del sondeo de opinión, describiendo sobre todo los pormenores de la muestra. Luego se presentan los resultados referidos al principal problema del país; el artículo continúa con un apartado dedicado a las opiniones sobre la economía. Después se abordan los resultados de las opiniones sobre la delincuencia. El último gran apartado se refiere a los político y éste se divide en dos partes: la evaluación del gobierno durante 1998 y las opiniones de los salvadoreños de cara a los comicios presidenciales de 1999. El artículo cierra resumiendo los puntos más importantes.

\section{Aspectos metodológicos}

La encuesta fue realizada entre los días 12 y 15 de diciembre de 1998 por el IUDOP con el objetivo de conocer cómo evalúan los salvadoreños la situación política, económica y social del país durante 1998; así como también indagar sobre el interés y la confianza que la población tiene en la política nacional y a la vez recoger preferencias partidistas de cara a las próximas elecciones.

El cuestionario empleado para llevar a cabo esta investigación se componía básicamente de cinco partes. En la primera se recogían los datos demográficos de la persona. En la segunda parte, se preguntaban sobre algunos hechos acaecidos en el país durante 1998 (por ejemplo, la elección del nuevo Procurador de Derechos Humanos, la Tormenta Tropical Mitch, el desempeño del gobierno, entre otras cosas). Había, además, un apartado dedicado a recoger las opiniones de los residentes del Área Metropolitana de San Salvador sobre algunos acontecimientos que les afectan (por ejemplo, las políticas de la Alcaldía de San Salvador, la consirucción de los pasos a desnivel, etc.). Las interrogantes de otro bloque estaban orientadas a recoger la percepción de los salvadoreños sobre cl tema de la seguridad pública y la situación de delincuencia en el país. La encuesta dedicaba también un espacio para preguntar por los medios de comunicación que, en términos de periódico y televisión, tienen una mayor circulación y audiencia. La última parte del cuestionario constaba de preguntas elaboradas para sondear la participación política ciudadana, sobre todo de cara a las elecciones de marzo de 1999.

La muestra nacional se diseñó de tal manera que reflejase lo más fielmente posible la totalidad de la población salvadoreña, esto según los datos del Censo Nacional de Población de 1992 y del informe disponible de la Encuesta de Hogares de Propósitos Múltiples de la Dirección de Información del Ministerio de Economía de 1996. La cuota muestral incluyó 40 municipios de los catorce departamentos de la República. En cada departamento, la muestra se dividió en dos grandes sectores: urbano y rural. El sector rural se dejó como una categoría aparte por las dificultades de encontrar criterios de tipilicación en cl campo. En cl muestreo se consideraron también cuotas por sexo y edad de los encuestados, de tal manera que cada boleta estaba marcada con las características que debía cumplir el encuestado para ser tomado en la 
investigación. La aplicación del cuestionario se hizo por aproximación no sistemática a los hogares ubicados en los municipios y las zonas definidas aleatoriamente. Los entrevistadores explicaban a las personas abordadas los objetivos y el tema de la encuesta. En cada caso se entrevistó únicamente a personas que quisieran contestar (una persona por hogar) y que cumpliera con los requerimientos descritos en la boleta para completar la cuota muestral.

La muestra final obtenida fue de 1,239 encuestas válidas, con un margen de error estimado de +/0.04 (cuatro por ciento). El 47.9 por ciento de los encuestados pertenece al sexo masculino y el 52.1 por ciento corresponde al sexo femenino. La edad promedio es 36.7 años con una desviación lípica de 14.3 años. Los entrevistados tienen un promedio general 7.9 años de estudio y el 51.6 por ciento de ellos se encuentran empleados en la actualidad o poseen un trabajo propio.

Los departamentos fueron agrupados en cinco zonas: zona occidental (Ahuachapán, Santa Ana y Sonsonate), zona central (área rural de La Libertad, departamento de Chalatenango y el área rural de San Salvador), zona metropolitana (área urbana de San Salvador y área urbana de Antiguo Cuscatlán y Nueva San Salvador), zona paracentral (Cuscatlán, Cabañas, San Vicente y La Paz) y zona oriental (Usulután, San Miguel, Morazán y La Unión). En el Cuadro 1 se presenta la distribución de las personas entrevistadas según estrato social y departamento en el cual residen.

Cuadro 1

Distribución de la población encuestada según departamento y sector social

\begin{tabular}{|c|c|c|c|c|c|c|c|c|c|}
\hline \multirow[b]{2}{*}{ Departamento } & & \multirow[b]{2}{*}{ Alto } & \multicolumn{3}{|c|}{ SECTOR SOCIAL } & \multirow[b]{2}{*}{ Marginal } & \multirow[b]{2}{*}{ Rural } & \multicolumn{2}{|c|}{ TODOS } \\
\hline & & & Medio-alto & Medio-bajo & Obrero & & & $\mathrm{N}$ & $\%$ \\
\hline \multirow[t]{2}{*}{ Todos } & $\%$ & 6.7 & 13.7 & 13.6 & 20.2 & 13.0 & 32.8 & - & 100.0 \\
\hline & $N$ & 83 & 170 & 168 & 250 & 161 & 407 & 1239 & - \\
\hline Ahuachapán & & 3.6 & 2.4 & 2.4 & 2.8 & 2.5 & 9.8 & 62 & 5.0 \\
\hline Santa Ana & & 8.4 & 7.6 & 7.7 & 8.0 & 8.1 & 10.8 & 110 & 8.9 \\
\hline Sonsonate & & 6.0 & 6.5 & 6.5 & 6.4 & 6.8 & 8.6 & 89 & 7.2 \\
\hline La Libertad & & 9.6 & 9.4 & 9.5 & 9.6 & 9.3 & 12.3 & 129 & 10.4 \\
\hline Chalatenango & & 3.6 & 2.4 & 2.4 & 2.8 & 2.5 & 5.2 & 43 & 3.5 \\
\hline San Salvador & & 37.3 & 39.4 & 38.1 & 37.2 & 37.9 & 11.5 & 363 & 29.3 \\
\hline Cabañas & & 2.4 & 1.8 & 1.8 & 2.0 & 2.5 & 4.2 & 34 & 2.7 \\
\hline Cuscatlán & & 2.4 & 4.1 & 3.0 & 3.2 & 3.1 & 3.7 & 42 & 3.4 \\
\hline San Vicente & & 2.4 & 2.4 & 2.4 & 2.4 & 2.5 & 3.4 & 34 & 2.7 \\
\hline La Paz & & 3.6 & 4.7 & 4.8 & 4.8 & 5.0 & 4.9 & 59 & 4.8 \\
\hline Usulután & & 4.8 & 5.3 & 6.5 & 5.2 & 5.0 & 8.6 & 80 & 6.5 \\
\hline San Miguel & & 7.2 & 8.2 & 8.9 & 8.4 & 8.7 & 5.2 & 91 & 7.3 \\
\hline Morazán & & 1.2 & 2.4 & 2.4 & 2.4 & 2.5 & 5.2 & 40 & 3.2 \\
\hline La Unión & & 7.2 & 3.5 & 3.6 & 4.8 & 3.7 & 6.6 & 63 & 5.1 \\
\hline
\end{tabular}

\section{Los problemas del país a finales de $\mathbf{1 9 9 8}$}

Como es usual en las encuestas de la UCA, sobre todo en aquéllas que tienen como objetivo evaluar el curso del año que finaliza, el sondeo realizado en diciembre de 1998 preguntó a los ciudadanos por los principales problemas del país. En este rubro, puede decirse que no hay cambios sig- nificativos, pero este hecho en sí mismo es muy elocuente con respecto a la poca movilidad de la opinión pública sobre las dificultades nacionales. En el Cuadro 2 se puede ver que las cosas que más preocupan a los salvadoreños son la delincuencia, la pobreza, el desempleo y la economía mencionada de manera genérica. En el fondo tales 
respuestas se refieren a dos grandes preocupaciones como ha sido siempre: violencia y economía. Hay, sin embargo, un movimiento importante en cuanto a los temas que prevalecen en el lado de lo económico. En el pasado, sobre todo, entre los años de 1995 y 1997, la inquietud ciudadana por el problema de la inflación —expresado popularmente como "la carestía", "el costo de la vida" o "el encarecimiento"- era mucho más elevada. Por ejemplo, la encuesta de evaluación de 1996 mostraba que casi el 8 por ciento de los salvadoreños veían a la inflación como el principal problema (ver IUDOP, 1997) y unos meses antes el porcentaje había rondado el 13 por ciento. En la actualidad, como lo demuestran los resultados expuestos, tal tendencia no va más allá del 3 por ciento; en su lugar, los aspectos económicos que están recibiendo más atención son la pobreza y el desempleo. Probablemente esto estaría relacionado con las expectativas de la gente con respecto a los planes de reducción de la pobreza y aumento de empleo expuestos por el gobierno y que no se han visto concretados hacia el final del ciclo presidencial.

De acuerdo con los resultados, los principales problemas del país no siguen una distribución par- ticularmente distinta según el estrato socioeconomico de los entrevistados -exceptuando en algunos casos-; de hecho, no hay una tendencia clara que indique que las personas más pobres se preocupan más por la pobreza o que las personas de mejor posición social estén significativamente más ansiosas por la criminalidad. Esto indica una opinión pública relativamente consistente en lo que se refiere a los problemas del país en las distintas clases o grupos socioeconómicos. Donde las diferencias parecen más notables es en la comparación de los resultados por partido político. Aunque la delincuencia es, sin duda, el problema particular más citado en cualquier grupo político, el peso que cada partido le atribuye no es siempre el mismo: partidos como ARENA y el Partido Demócrata Cristiano señalan más el problema de la criminalidad que el resto, en tanto que los simpatizantes del recientemente formado Centro Democrático Unido muestran el nivel más bajo de preocupación por el crimen. Por su parte, la pobreza destaca entre los partidos de izquierda o afines a la izquierda (FMLN y CDU), y el desempleo sobresale entre los ciudadanos que no respondieron a la pregunta de la intención de voto.

\section{Cuadro 2}

Principal problema del país según variables (En porcentajes)

\begin{tabular}{|c|c|c|c|c|c|c|c|c|}
\hline \multirow[b]{2}{*}{ Variables } & \multirow[b]{2}{*}{ Delincuencia } & \multirow[b]{2}{*}{ Pobreza } & \multicolumn{3}{|c|}{ PRINCIPAL PROBLEMA } & \multirow[b]{2}{*}{ Mal gobierno } & \multirow[b]{2}{*}{ Otras respuestas } & \multirow{2}{*}{$\begin{array}{l}\text { No } \\
\text { sabe }\end{array}$} \\
\hline & & & Desempleo & Economía & Inflación & & & \\
\hline Todos & 43.7 & 19.9 & 15.1 & 9.0 & 2.7 & 2.3 & 4.9 & 2.5 \\
\hline \multicolumn{9}{|l|}{ Estrato } \\
\hline Alto & 49.4 & 14.5 & 13.3 & 10.8 & 0.0 & 6.0 & 4.8 & 1.2 \\
\hline Medio-alto & 44.7 & 23.5 & 14.1 & 8.8 & 0.6 & 3.5 & 4.7 & 0.0 \\
\hline Medio-bajo & 46.4 & 17.3 & 12.5 & 10.1 & 1.8 & 2.4 & 8.3 & 1.2 \\
\hline Obrero & 40.0 & 18.8 & 19.6 & 12.8 & 1.6 & 2.4 & 3.2 & 1.6 \\
\hline Marginal & 45.3 & 15.5 & 14.9 & 6.8 & 5.0 & 3.1 & 4.3 & 5.0 \\
\hline Rural & 42.5 & 22.9 & 14.3 & 6.9 & 4.2 & 0.5 & 4.9 & 3.9 \\
\hline Ninguno & 39.6 & 18.4 & 14.6 & 9.9 & 3.8 & 2.4 & 8.5 & 2.8 \\
\hline ARENA & 47.0 & 20.8 & 12.9 & 7.6 & 1.7 & 1.9 & 6.0 & 2.1 \\
\hline FMLN-USC & 39.8 & 22.8 & 15.8 & 10.5 & 2.9 & 4.1 & 3.5 & 0.6 \\
\hline $\mathrm{CDU}$ & 29.3 & 29.3 & 14.6 & 9.8 & 2.4 & 4.9 & 9.8 & 0.0 \\
\hline PDC & 46.9 & 9.4 & 9.4 & 18.8 & 6.3 & 6.3 & 0.0 & 3.1 \\
\hline Voto secreto & 41.7 & 20.8 & 14.6 & 14.6 & 4.2 & 2.1 & 0.0 & 2.1 \\
\hline Otros & 40.9 & 13.6 & 9.1 & 9.1 & 4.5 & 9.1 & 9.1 & 4.5 \\
\hline No sabe, no responde & 46.3 & 18.0 & 19.4 & 7.5 & 2.4 & 0.3 & 2.0 & 4.1 \\
\hline
\end{tabular}

En su opinión, ¿cuál es el principal problema que enfrenta actualmente El Salvador? 
Con todo, es interesante advertir que a pesar de los cambios que han tomado lugar en el país, la percepción básica de los ciudadanos sobre los problemas fundamentales sigue siendo la misma. La preeminencia de los aspectos de seguridad pública y económicos en el pensamiento ciudadano por sobre otras problemáticas que tienen relevancia actual como la corrupción, el medioambiente, el transporte público, etcétera, muestra la importancia que la población suele darle a aquellos problemas a lo largo del tiempo. De ahí que cuando se evalúa la gestión del gobierno, usualmente los temas que se le achacan como fracasos son los mismos por los cuales se inquietan los salvadoreños.

\section{La situación económica}

Uno de los datos más interesantes del sondeo de la UCA es el que recoge las opiniones ciudadanas sobre el cambio de las condiciones económicas durante 1998. Estas opiniones muestran que el punto más sensible sobre economía es, en buena medida, la pobreza, y que existe una opinión predominante de que las condiciones económicas no mejoran.

Concretamente, dos de cada tres salvadoreños consideran que la pobreza aumentó durante 1998, mientras que el resto de la población se dividió en afirmar que la pobreza se ha mantenido igual o ha disminuido. La opinión sobre el aumento de la pobreza es sostenida por aquellas personas que hahitan en la zona metropolitana de San Salvador, por los que se ubican en los estratos altos y medio-bajos, por las personas de mayor edad y por los sujetos con mayor nivel de educación. En términos de opción partidista, la opinión sobre un crecimiento de la pobreza en el país es sostenida con más intensidad por las personas que rechazan tener un partido de preferencia, por los volantes del FMLN y por los votantes del Partido Demócrata Cristiano.

Esla percepción sobre el aumento de la pobreza está vinculada a otras percepciones sobre las condiciones económicas. Un poco más de la mitad de los salvadoreños, el 51.7 por ciento, opinaron que la cconomía del país se mantuvo igual durante el año que termina; una tercera parte, el 34.5 por
Figura 1

Opinión sobre la pobreza en el país durante 1998 en comparación con años anteriores

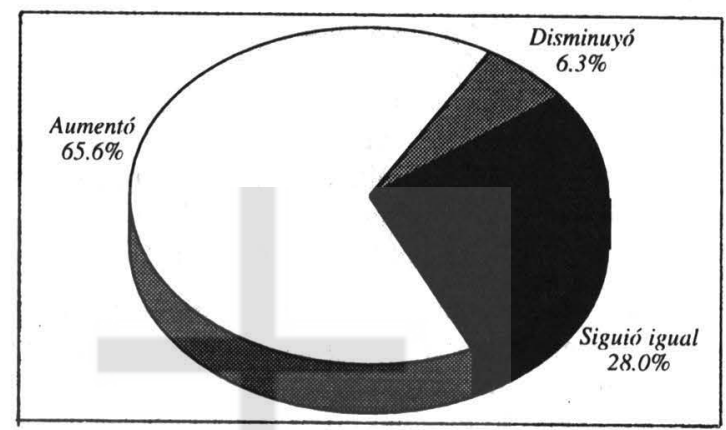

¿Cree usted que durante este año, la pobreza en el país aumentó, siguió igual o disminuyó?

ciento, sostuvo que empeoró y sólo un 12.7 por ciento. En el nivel más doméstico, las opiniones fueron menos críticas: el 61.2 por ciento de los consultados sostuvo que su situación económica familiar se ha mantenido igual en 1998 con respecto al pasado, el 24.4 por ciento piensa que la misma empeoró, y el 14.4 por ciento dijo que su siluación doméstica había mejorado. Lo anterior muestra que las personas consideran que la economía del país no ha avanzado de la misma forma en la que han avanzado sus economías particulares. De hecho, la opinión de que la situación económica se ha mantenido igual no parece ser una afirmación positiva, en el sentido de que está igual de bien que antes, sino que tiene cierto sentido negativo, pues las cosas no parecen haber cambiado para mejorar. La gente que dijo que la pobreza ha aumentado en el país, tendió a responder que la economía del país y la economía familiar se han mantenido igual o han empeorado.

Esta tendencia de opinión sin embargo, parece ser menos crítica que la correspondiente a la evaluación de 1997, cuando el sondeo en cuestión mostraba que la gente se decantaba un poco más en las 
respuestas de que las condiciones habían empeorado. Aunque esto no ha sido un cambio radical en los resultados con respecto a la evaluación de 1997 , las tendencias sugieren que la preocupación por lo económico es cada vez menos aguda en comparación con años anteriores. Efectivamente, como puede verse en la Figura 2, entre 1995 y 1996 la mayoría de los salvadoreños pensaba que sus condiciones económicas - tanto en el ámbito nacional como en el ámbito familiar- habían empeorado; sin embargo, esta opinión disminuye de forma sustancial hacia 1997 y continúa el descenso en 1998.

Esto sugiere que, como se ha dicho anteriormente, buena parte de los ciudadanos no parecen estar tan preocupados por la situación económica del país como lo estaban en el pasado. Lo anterior no significa que no exista un nivel de preocupación importante y válido de la ciudadanía por la situación económica; más bien, muestra que a finales de 1998 - a pocos meses de finalizar la gestión presidencial de Calderón Sol y, sobre todo, a poco tiempo de celebrarse las elecciones- esta preocupación no tiene la magnitud que tuvo a mediados del ciclo presidencial inaugurado en el 94. Este es un fenómeno interesante, pues estaría vinculado con el movimiento de las preferencias de los ciudadanos $\longrightarrow$ de una parte de éstos- a ciertos partidos políticos. Otros trabajos han mostrado la importancia de la opinión pública en la configuración de los apoyos políticos ciudadanos (Cruz, 1997), y el hecho de que los ciudadanos no están tan angustiados por la economía podría estar favoreciendo la imagen del gobierno y del partido en el mismo al final de su mandato.

\section{Figura 2}

Tendencias de las opiniones de que las condiciones económicas han empeorado

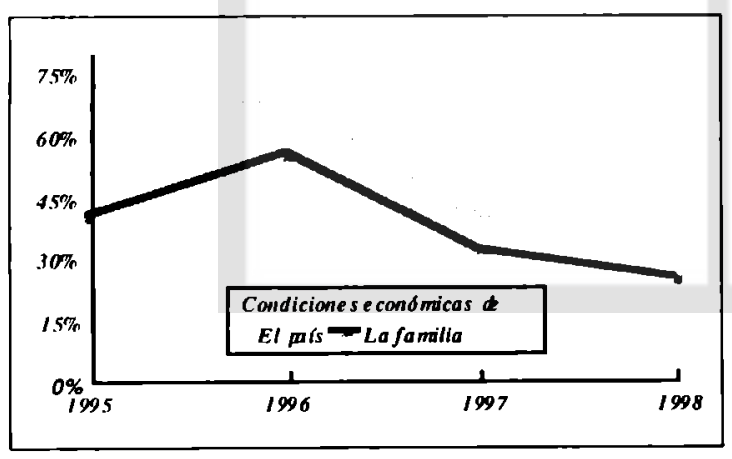

Fuente: IUDOP, Serie de informes.
Esta disminución en las opiniones críticas sobre la economia puede parecer contradictoria con uno de los resultados expresados anteriormente, el del aumento de la pobreza. La explicación estaría en el hecho de que cuando la gente habla de pobreza no se refiere a sus propias condiciones de vida, prueba de ello sería el dato descrito arriba de que quienes más sostienen esa opinión son los ciudadanos de clases medias y altas y no los más pobres; cuando la gente habla de aumento de pobreza probablemente se basa en la percepción de que no ha habido un combate efectivo de la misma, y que el problema tiene tanta o más gravedad que en el pasado.

Además y volviendo al tema del cambio de opiniones sobre el problema económico, no puede dejar de advertirse que esta tendencia sobre la economía podría estar asociada con el descenso en la preocupación por el costo de la vida, esto es, por la inflación. Como ya se ha apuntado, 1998 se caracterizó por ser un año en el que los salvadoreños se preocuparon comparativamente menos por el costo de la canasta básica y este aspecto, que suele ser muy sensible en la percepción pública, podría estar condicionando algunas de las reacciones sobre la economía.

\section{La delincuencia}

El otro gran tema que preocupa a los salvadoreños, y que sin duda dominó la opinión pública durante 1998, es el de la criminalidad. La encuesta de la UCA revela que, en este caso, el pensamiento que prevalece entre los ciudadanos es que la delincuencia aumentó durante 1998: el 66 por ciento de los consultados dijo que el crimen había aumentado en el año en cuestión, en tanto que el 23.8 por ciento dijo que los niveles de crimen se habían mantenido igual y sólo el 9.4 por ciento fue de la opinión que la violencia criminal disminuyó.

Según la encuesta, la idea de que el crimen ha aumentado se encuentra con más frecuencia en la zona central del país (los departamentos de San Salvador, La Libertad y Chalatenango) y especialmente en el área metropolitana de San Salvador; se encuentra también con más reiteración entre las personas que pertenecen a los estratos medios, entre los que tienen más años de formación educativa y entre las personas que votarían por el FMLN o que no votarían por partido alguno. Por el otro lado, es interesante ver que las opiniones sobre disminución de la delincuencia son más frecuentes 
en el área rural del país, en los departamentos al oriente de San Salvador y entre las personas que no definen su voto de preferencia y las que votarían por ARENA.

Sin embargo, la percepción sobre el aumento de la delincuencia durante 1998 no parece depender siempre de las propias experiencias con respecto a los hechos criminales. La encuesta del IUDOP preguntó a los ciudadanos si habían sido víctimas directamente de algún hecho delincuencial durante 1998, y el 21.2 por ciento contestó afirmativamente. Sin embargo, esta proporción no se mantuvo igual para todas las personas agrupadas según distintas variables. Por ejemplo, en el área metropolitana de San Salvador, el porcentaje de victimización se eleva al 26.6 por ciento y entre las personas del sector medio-alto llega hasta el 35.3 por ciento, más del doble que el registrado por sus compatriotas que habitan en el área rural. Un resultado que llama la atención es el que señala que entre los partidos existen diferencias en la victimización de sus correligionarios: los simpatizantes del Partido Demócrata Cristiano muestran un nivel de victimización particularmente alto y distinto del resto de grupos. Esta diferencia podría deberse, de alguna manera, a la configuración del perfil del votante del PDC, en el sentido de que algunas características del mismo (zona donde reside, estrato, tipo de ocupación) lo hacen más propenso a sufrir por la delincuencia. No obstante, hay que decir que la encuesta no ofrece herramientas adicionales para explicar esas diferencias en la victimización.

En todo caso, el Cuadro 3 revela que la percepción sobre el aumento de la delincuencia no siempre es más alto entre las personas que han sufrido algún evento criminal, pero hay coincidencias importantes en los habitantes del área metropolitana de San Salvador y en los procedentes de la clase media-alta; en ambos casos, el porcentaje de opinión y de victimización es más alto que en el resto de grupos.

Empero, el dato más elocuente respecto a la discrepancia entre opinión y realidad desde los resultados de las encuestas es el que se obtiene cuando se comparan los niveles de victimización de 1998 con el año anterior. En 1997, el porcentaje de personas que afirmó haber sido víctima de un delito fue del 27.5 por ciento (ver IUDOP, 1998a), más elevado que el consignado en este sondeo. De hecho, otras encuestas más especiali-

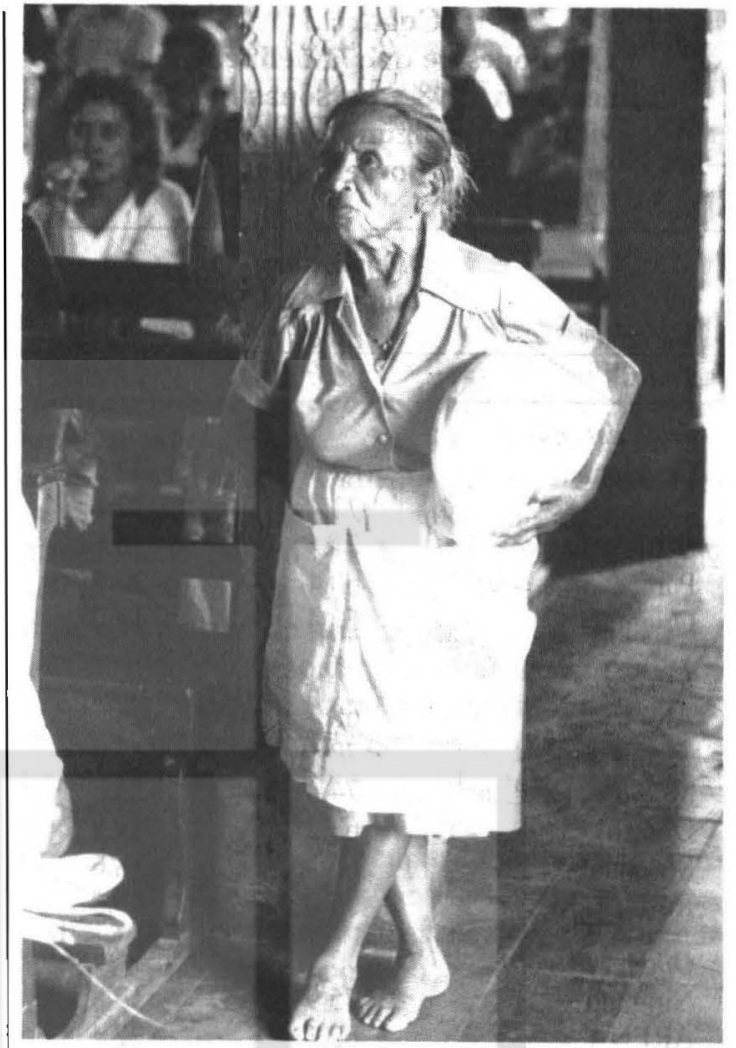

zadas sobre el tema han confirmado esa tendencia de disminución de los registros de victimización desde 1996 (IUDOP, 1998b), pero eso no pasa con las opiniones respecto a la delincuencia. Las respuestas a la encuesta señalan que en 1998, la gente estuvo más preocupada por el problema y que a pesar de los registros, percibieron que el problema delincuencial aumentó de manera significativa.

\section{Lo político}

\subsection{La evaluación del gobierno}

Tradicionalmente, el IUDOP ha dedicado unas cuantas preguntas para evaluar la gestión del gobierno en las encuestas de fin de año, esto se ha hecho además del estudio monográfico que sobre el mismo tema hace el instituto a mediados de cada año. Ese seguimiento permite tener una idea de cómo se han movido las opiniones ciudadanas con respecto a la gestión del gobierno y contrastar las mismas con el resto de conceptos sobre el país. Esta pesquisa no es la excepción. A los entrevistados se les pidió que identificaran los logros y los 


\section{Cuadro 3 \\ Opiniones de que la delincuencia aumentó en 1998 y personas que fueron víctimas de algún hecho delictivo (En porcentajes)}

\begin{tabular}{lcc}
\hline Variables & $\begin{array}{c}\text { Opinión de } \\
\text { aumento de } \\
\text { crimen }\end{array}$ & $\begin{array}{c}\text { Víctimas } \\
\text { en } 1998\end{array}$ \\
\hline Todos & 66.0 & $2 l .2$ \\
\hline Zona del país & & \\
Occidental & 62.5 & 22.2 \\
Central & 71.4 & 17.1 \\
Metropolitana & 71.4 & 26.6 \\
Paracentral & 60.4 & 20.7 \\
Oriental & 62.4 & 15.0 \\
Estrato & & \\
Alto & 68.7 & 25.3 \\
Medio-alto & 77.1 & 35.3 \\
Medio-bajo & 74.4 & 25.6 \\
Obrero & 63.2 & 19.2 \\
Marginal & 66.5 & 18.6 \\
Rural & 59.0 & 15.0 \\
Opción de voto & & \\
Ninguno & 75.9 & 19.8 \\
ARENA & 62.1 & 22.4 \\
FMLN-USC & 76.6 & 25.7 \\
CDU & 73.2 & 22.0 \\
PDC & 68.8 & 40.6 \\
Voto secreto & 54.2 & 14.6 \\
Otros & 68.2 & 13.6 \\
No sabe, no responde & 58.8 & 17.3 \\
\hline
\end{tabular}

¿Ha sido usted victima de algún hecho delincuencial durante 1988? / En comparación con el año pasado, ¿en este año la delincuencia aumentó, siguió igual o disminuyó?

fracasos del gobierno en el año, al mismo liempo que se les pidió que asignaran una nola - en una escala de 0 a 10 - para calificar la gestión gubernamental.

Entre los logros señalados al gobierno durante 1998 están: el arreglo de la infraestructura (21.5 por ciento), el esfuerzo de la reforma educativa ( 12.5 por ciento), "la construcción de los pasos a desnivel" (8.6 por ciento), el mejoramiento de los servicios públicos (4.4 por ciento), la ayuda a los pobres (3.8 por ciento), la política económica (3.5 por ciento) y el combate contra la delincuencia ( 2 por ciento), entre otros aspectos señalados. No obstante, casi la tercera parte de los consultados (32 por ciento) sostuvo que el gobierno actual no tiene logro alguno identificable y cerca del 7 por ciento prefirió no responder a la pregunta. En el fondo, si se suman las respuestas "arreglo de la infraestructura" y "pasos a desnivel" se tiene que el aspecto positivo más señalado del gobierno es el de la infraestructura: tres de cada diez salvadoreños lo mencionan. Aún así, la respuesta más frecuente sigue siendo "ningún logro". Sin embargo, examinando estas opiniones en perspectiva, se tiene que en esta ocasión es cuando más se han logrado identificar aspectos positivos de la gestión presidencial de Calderón Sol; en el pasado, alrededor del 60 por ciento de la población no identificaba logro alguno y el aspecto más citado no llegaba siquiera al 20 por ciento (IUDOP, 1997).

En la otra cara de la moneda, los salvadoreños acordaron en señalar a los problemas principales del país como los fracasos del gobierno en el año que finaliza. El incremento de la delincuencia (22 por ciento), la falta de empleos (20.1 por ciento), la atención a los "ricos" -o que es lo mismo: la desatención a los pobres- (11.1 por ciento) y la política económica (7.1 por ciento), figuran como las fallas citadas con más frecuencia entre varias mencionadas. En este caso, un poco más del 11 por ciento dijo que no encontraba ningún fracaso del gobierno y otro 11 por ciento no respondió a la pregunta planteada, lo que significa que el 22 por ciento de los consultados no identificaron fallas a la gestión del segundo gobierno de ARENA durante 1998.

La concordancia entre la identificación de los principales problemas del país y los fracasos del gobierno sugiere, como ha sido evidenciado en estudios anteriores, que buena parte de la población sigue achacando al gobierno la existencia de los problemas nacionales y que su falta de solución sigue siendo su principal debilidad.

No obstante, en términos generales, la imagen del gobierno ha mejorado durante 1998. La nota promedio de calificación del desempeño del gobierno durante 1998 fue de 5.7, lo que se constituye en el mejor promedio obtenido por la gestión de Calderón Sol desde sus primeros cien días de gobierno. Efectivamente, si se examinan las tendencias de calificación del trabajo del gobierno, se podrá ver que los datos obtenidos a finales de 
1998 representan una separación leve pero importante de la tendencia mostrada en la mayor parte de su mandato (ver Figura 3). La calificación no es en sí misma positiva, pero es claramente menos negativa que la media observada durante la mayor parte del mandato. La Figura 3 muestra que la opinión sobre el gobierno desciende significativamente luego del primer año de mandato, se mantiene en esos niveles en la mayor parte del tiempo (por más de dos años) y hacia diciembre de 1997 comienza a registrar una recuperación que se incrementa en la segunda mitad de 1998. Así, seis meses antes de concluir su labor, la administración de Calderón obtiene la mayor nota de evaluación desde sus primeros días, sin que ella sea particularmente positiva. Estos resultados son coherentes con las tendencias de opinión con respecto a los logros y los fracasos del gobierno.

\section{Figura 3}

Tendencia en la calificación del trabajo del gobierno de Calderón Sol desde 1994

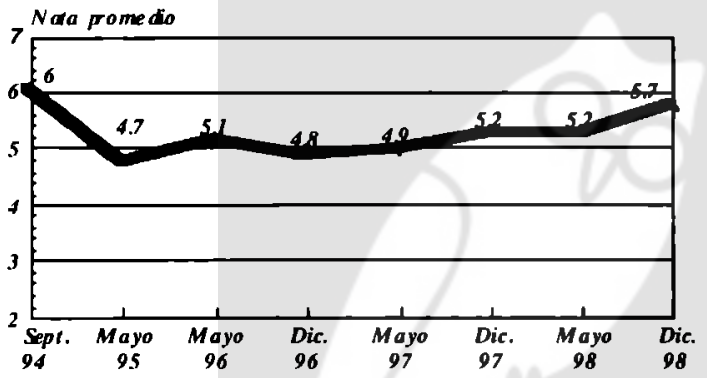

Fuente: IUDOP, Serie de informes.

Ahora bien, ¿a qué se deben esos movimientos en el juicio público sobre la presidencia? Pueden haber muchos factores - percepción sobre el país, la propaganda de imagen del gobierno en los medios, la construcción de los pasos a desnivel en la capital- pero probablemente uno de los que más estarían incidiendo es la campaña electoral que se ha iniciado desde 1998 con vistas a los comicios presidenciales de 1999. La proximidad de las elecciones del 99 y el esfuerzo de la campaña electoral, orquestado por el partido gobernante, han implicado que el gobierno cuide muy bien sus pasos en la última etapa de su gestión y que invierta considerables recursos en mejorar su imagen. La construcción simultánea de varios pasos a desnivel en la ciudad, iniciada a ocho meses de terminar la gestión, es una muestra palpable de este esfuerzo por incidir en su propia imagen pública. Sin duda, ello - la imagen del gobiemo al terminar su período- tendrá también cierto impacto en la determinación de los resultados electorales del año 99.

\subsection{Las elecciones de 1999}

En el ámbito de lo político, uno de los aspectos que más exploró la encuesta fue el movimiento de las preferencias político-partidistas de cara a los comicios presidenciales de 1999. La encuesta del IUDOP, realizada a mediados del mes de diciembre, registró una serie de datos interesantes. En primer lugar, mostró que buena parte de la población no tiene interés y confianza en el proceso electoral, ello a pesar de que las entrevistas fueron hechas cuando la campaña electoral tenía al menos un mes de haber arrancado. En segundo lugar, mostró que la reciente postulación de Zamora como candidato del nuevo partido Centro Democrático Unido (CDU) fue recibida por un sector - aunque pequeño- de la población. Además, la encuesta mostró que a un poco más de dos meses de las elecciones, ARENA reúne muchas más intenciones de voto que cualquier otro partido político.

\subsubsection{Las actitudes ante las elecciones}

Como parece ser la norma en los últimos eventos electorales, el sondeo encontró que una porción importante de la ciudadanía no está muy motivada por los comicios presidenciales. Según la Figura 4, un poco más del 55 por ciento de los salvadoreños tienen poca o ninguna confianza en el proceso eleccionario, el 27.4 por ciento tiene algo de confianza y sólo el 16.2 por ciento tiene mucha confianza en el proceso electoral. Algo parecido sucede con el interés mostrado por las elecciones: el 62 por ciento de los entrevistados dijo que no tenía interés o que tenía poco interés por los comicios a celebrarse el 7 de marzo de 1999, mientras que el 20.2 por ciento señaló algo de interés y el 17.5 por ciento expresó mucho.

Lo anterior sugiere que la mayoría de los salvadoreños no se han sentido muy motivados por la campaña electoral desarrollada mientras se hacía la encuesta, ni se han sentido muy confiados hacia los comicios. Es interesante ver que prevalece un poco más de desinterés que de desconfianza, lo cual no ha sido siempre así en eventos anteriores monitoreados por los sondeos de la UCA. En todo 
Figura 4

Niveles de interés y confianza en las elecciones

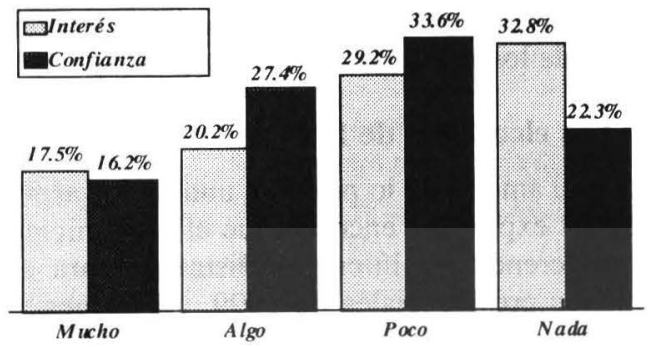

¿El próximo proceso electoral le interesa mucho, algo poco o nada?' ¿Cuánta confianza tiene el próximo proceso electoral?

caso, ambas actitudes usualmente están presentes entre las personas que deciden abstenerse o que no votan en los eventos electorales. Por ejemplo, un poco más del 85 por ciento de las personas que dijeron que no votarán por partido alguno mostraron poca o ninguna confianza en las elecciones, y el 88 por ciento mostró poco o ningún interés. En tal sentido, estos datos estarían apuntando a que un porcentaje importante de ciudadanos no asistiría a votar.

La misma encuesta ofrece otros indicadores sobre el asunto. Preguntados directamente sobre el interés por asistir a votar, el 28.6 por ciento registró el interés más alto, el 22.1 por ciento dijo que tenía "algo" de interés, el 26 por ciento mencionó que tenía "poco" y el 23.3 por ciento dijo "nada". Con tales resultados sólo se puede tener la certeza de que al menos alrededor del 29 por ciento va a votar, en el resto de ciudadanos existe cierto grado de incertidumbre que dificulta una proyección de la participación ciudadana a partir de estos datos. En todo caso, hay más razones para pensar que el porcentaje de abstencionismo será de nuevo bastante alto, probablemente no menor del 49 por ciento (esto si se suman los porcentajes de las personas que dijeron que tienen poco o ningún interés por acudir a las urnas).

\subsubsection{La imagen de los candidatos y la inten- ción de voto por partido}

Uno de los aspectos más importantes en el curso de una elección presidencial lo constituye la imagen de los candidatos que están compitiendo por la presidencia, esto es, cómo la gente percibe a las personas que están postuladas a ocupar la silla del Ejecutivo y qué tanta aceptación tienen entre el público. Para establecer qué candidato, en tanto que imagen personal, goza con más simpatías entre la población, el sondeo del IUDOP pidió a los ciudadanos que dieran su opinión sobre los aspirantes presidenciales de dos maneras. En primer lugar, por cada uno de los candidatos se preguntó si lo conocían o habían oído hablar de él; si la respuesta era positiva, se pedía que dieran su opinión sobre el mismo asignándole una nota de una escala de 0 a 10 . En segundo lugar, se preguntó directamente a los ciudadanos por la persona que elegirían de entre los postulados oficialmente para la presidencia. Los resultados de ambos métodos fueron muy similares.

Los resultados del primer método se presentan en el Cuadro 4. Como puede verse, Francisco Flores resulta ser el candidato más conocido por la gente y con la calificación más alta, le siguen Facundo Guardado y Rubén Zamora con porcentajes de reconocimiento arriba del 70 por ciento y con una calificación empatada. Luego de ellos existe una gran brecha en el conocimiento del resto de candidatos, los cuales obtienen a su vez las calificaciones más bajas. Existe un fenómeno acá que es importante mencionar. Los aspirantes presidenciales con mejor promedio de calificación son aquéllos que son más conocidos; pareciera que en la medida en que un candidato es menos conocido o reconocido por la población, en esa medida la opinión que la gente tiene de él es más pobre. Así, las cifras indicarían que un candidato no sólo tiene que ser conocido para que la gente pueda elegir por él, sino que también para que pueda acumular un caudal significativo de simpatías. 


\section{Cuadro 4 \\ Conocimiento y evaluación de los candidatos presidenciales}

\begin{tabular}{lcc}
\hline Candidato & $\begin{array}{c}\text { \% que lo conoce } \\
\text { o ha oído de él }\end{array}$ & $\begin{array}{c}\text { Nota } \\
\text { promedio }\end{array}$ \\
\hline Francisco Flores & 86.8 & 6.9 \\
Facundo Guardado & 78.3 & 5.6 \\
Rubén Zamora & 75.0 & 5.6 \\
Rodolfo Parker & 42.8 & 5.2 \\
Hernán Contreras & 35.6 & 5.0 \\
Nelson García & 15.3 & 4.7 \\
Francisco Ayala de Paz & 14.8 & 4.8 \\
\hline
\end{tabular}

A continuación le voy a mencionar a los candidatos presidenciales; ine gustaría que me dijera para cada uno de ellos si los conoce y cómo los evaluaría en una escala de 0 a 10.

Por otro lado, cuando se pidió a los ciudadanos que eligieran a uno de entre los aspirantes, los re- sultados muestran unas tendencias parecidas. El 41.4 por ciento escogió a Francisco Flores, el candidato de ARENA, el 11.5 por ciento se decidió por Rubén Zamora del Centro Democrático Unido, un porcentaje similar estadísticamente se decantó por Facundo Guardado del FMLN, el 3.1 por ciento eligió a Rodolfo Parker y el resto de resultados se dirigió a otros candidatos. Algo más del 30 por ciento no escogió a ninguno de los postulantes, ya sea porque no le convencen o porque prefirió no responder a la pregunta. Lo anterior señala varias cosas. Primero, que el candidato del partido oficial tiene una muy buena imagen entre los salvadoreños, por encima de las opiniones del resto de candidatos. Segundo, que Rubén Zamora recoge un porcentaje importante de simpatías que, en términos personales y no de partido, le hace disputar la segunda posición a Facundo Guardado. Tercero que el resto de candidatos no logran atraer por sí mismos apoyos importantes de la población.

\section{Cuadro 5}

Candidato a quién el encuestado elegiría para presidente del país según variables

$$
\text { (En porcentajes) }
$$

\begin{tabular}{lccccccc}
\hline Opción de voto & \multicolumn{7}{c}{ CANDIDATOS } \\
& Ninguno & $\begin{array}{c}\text { Francisco } \\
\text { Flores }\end{array}$ & $\begin{array}{c}\text { Rubén } \\
\text { Zamora }\end{array}$ & $\begin{array}{c}\text { Facundo } \\
\text { Guardado }\end{array}$ & $\begin{array}{c}\text { Rodolfo } \\
\text { Parker }\end{array}$ & Otros & $\begin{array}{c}\text { No } \\
\text { responde }\end{array}$ \\
\hline Todos & 14.5 & $4 I .4$ & $I I .5$ & 11.3 & 3.1 & 2.0 & $16 . I$ \\
\hline Ninguno & 50.0 & 19.8 & 10.4 & 7.5 & 1.9 & 1.4 & 9.0 \\
ARENA & 1.0 & 91.2 & 1.2 & 0.7 & 1.0 & 0.7 & 4.3 \\
FMLN-USC & 5.3 & 2.3 & 29.2 & 58.5 & 2.3 & 0.6 & 1.8 \\
CDU & 0.0 & 0.0 & 92.7 & 4.9 & 0.0 & 2.4 & 0.0 \\
PDC & 12.5 & 3.1 & 9.4 & 9.4 & 56.3 & 0.0 & 9.4 \\
Voto secreto & 12.5 & 16.7 & 8.3 & 2.1 & 0.0 & 8.3 & 52.1 \\
Otros & 4.5 & 22.7 & 4.5 & 4.5 & 4.5 & 45.5 & 13.6 \\
No sabc, no responde & 17.0 & 24.1 & 6.5 & 4.8 & 2.7 & 1.0 & 43.9 \\
\hline
\end{tabular}

Si usted uniera que escoger entre las personas que le mencioné, ¿a quién elegiría como presidente?

Al tomar estos resultados sobre el candidato y cruzarlos con las intenciones de voto por partido político, se obtienen algunas pistas importantes de cómo se mueven las simpatías de los institutos y de las personas políticas. Según el Cuadro 5, casi la totalidad, el 91.2 por ciento, de los areneros eligen a Flores y el resio de electores que quedan se distri- buyen de manera similar en el resto de candidatos. En el caso del Frente, no todos los electores del partido de izquierda se quedan con su candidato, $\mathrm{Fa}$ cundo Guardado, un porcentaje importante escoge a Zamora; mientras que éste es elegido por gran mayoría de los simpatizantes del CDU. Otro dato importante es el que se refiere a los electores de 


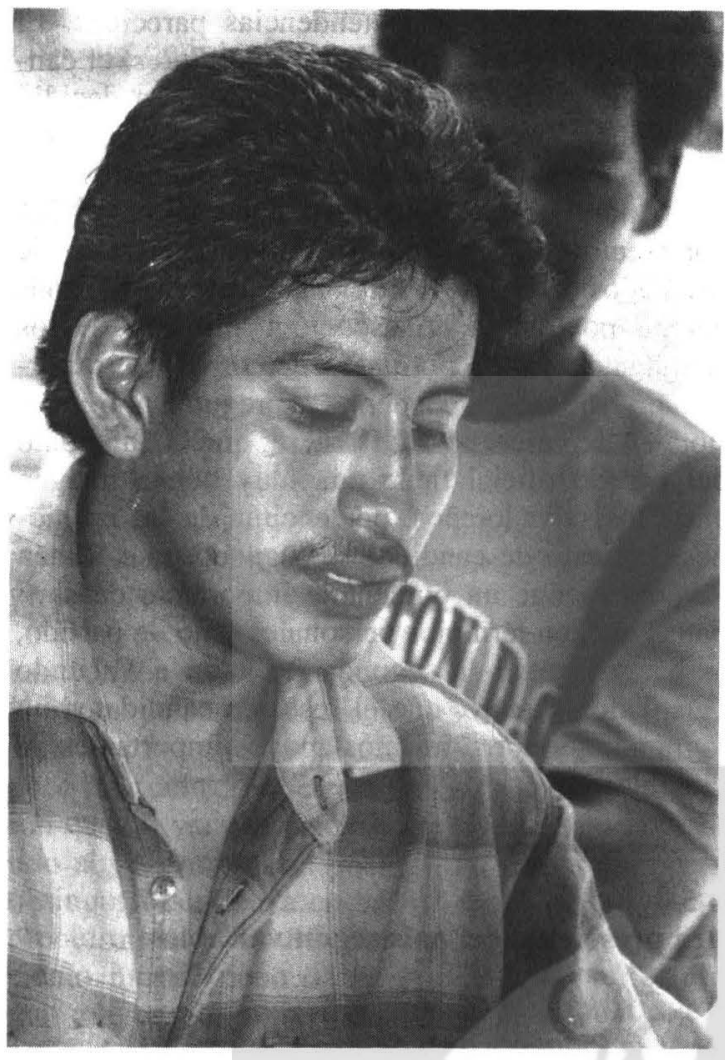

otros partidos, en este caso alrededor del 50 por ciento escoge a su candidato, pero el resto se decanta por las candidaturas de Flores, Guardado y Zamora.

Las cifras anteriores indican que no siempre las preferencias partidistas se correlacionan con las simpalías por los candidatos; porcentajes importantes de electores pueden preferir a otra candidatura, lo cual podría tener un efecto adverso para el partido mismo que no logra conjugar ambos elementos. De hecho, los resultados de las intenciones de voto revelan una orientación en algunos aspectos distinta a la mostrada por los candidatos.

De acuerdo con los resultados del sondeo realizado a mediados de diciembre de 1998, una tercera parte de los salvadoreños, el 33.8 por ciento, votarían por ARENA, el 13.8 por ciento votaría por el FMLN, un 3.3 por ciento preferiría al CDU y un 4.4 por ciento votaría por el resto de partidos políticos. Sin embargo, más del 40 por ciento de los consultados no expresaron su intención de voto ya sea porque no les gusta ningún partido, porque no saben por quién votar, o porque no lo quisieron manifestar al encuestador (voto secreto).

Como es usual, no toda la gente elige a cada uno de los partidos de la misma forma. ARENA ve incrementado su apoyo en ciertas zonas del interior del país, en los estratos altos y medio-altos; entre las mujeres, los jóvenes y los de mayor nivel de formación educativa. Por su parte, los electores de FMLNUnión se encuentran con más frecuencia entre los habitantes del área metropolitana de San Salvador, entre los pertenecientes al estrato medio-bajo; entre los hombres, los de mayor edad y los más instruidos.

Según las cifras, ARENA mantendría una ventaja notable sobre el segundo lugar ocupado por el FMLN. Sin embargo, la encuesta ya no es contundente cuando se trata de definir el tercer lugar dados los bajos porcentajes de preferencia del resto de partidos. Numéricamente, el CDU aparece en tercer lugar, pero sólo a unas escasas décimas de porcentaje del PDC -el tercer lugar tradicional desde 1994-, el resto de partidos obtienen porcentajes mínimos pero no muy lejos del CDU y el Partido Demócrata Cristiano. En tal sentido y considerando la acción del margen de error muestral a este nivel (+/- 4 por ciento), el posicionamiento del CDU en el tercer lugar es muy precario. Es importante, sin embargo, hacer notar que esta aparición del CDU en las preferencias políticas probablemente se deba a un efecto originado por su candidato - $\sin$ duda más conocido que el propio partido al momento de la encuesta' - y no tanto por el partido en sí mismo. En el resto de partidos pequeños, la relación entre candidato y partido parece ser la inversa: el partido es más conocido que el personaje.

Pero volviendo al tema de las intenciones de voto, ¿cómo explican los salvadoreños sus propias preferencias políticas? La encuesta abrió el espacio para que los entrevistados explicaran las razones por las cuales piensan votar por cada uno de los partidos. En el caso de ARENA, las razones más comunes expuestas por sus seguidores son: "porque podría hacer algo" ( 24.6 por ciento) y por el candidato (21.2 por ciento). La coalición FMLN-Unión recogió razones que señalan la necesidad del cambio ( 36.3 por ciento) y "porque po-

I. De hecho, el Centro Democrático Unido (CDU) se formó y presentó a la sociedad política salvadoreña pocos días antes de iniciar el trabajo de campo de este sondeo. 
Cuadro 6

Partido político por el cual votarian los encuestados

en diciembre de 1998 según variables

(En porcentajes)

\begin{tabular}{|c|c|c|c|c|c|c|c|c|}
\hline Variables & Ninguno & ARENA & $\begin{array}{r}\text { PARTI } \\
\text { FMLN-USC }\end{array}$ & $\begin{array}{l}\text { DO POI } \\
\text { CDU }\end{array}$ & $\begin{array}{l}\text { ПICO } \\
\text { PDC }\end{array}$ & $\begin{array}{l}\text { Voto } \\
\text { secreto }\end{array}$ & Otros & $\begin{array}{c}\text { No } \\
\text { responde }\end{array}$ \\
\hline Todos & 17.1 & 33.8 & 13.8 & 3.3 & 2.6 & 3.9 & 1.8 & 23.7 \\
\hline \multicolumn{9}{|l|}{ Zona del país } \\
\hline Occidental & 14.2 & 36.0 & 10.7 & 3.1 & 1.5 & 5.7 & 1.1 & 27.6 \\
\hline Central & 13.6 & 28.6 & 14.3 & 1.4 & 1.4 & 2.9 & 2.1 & 35.7 \\
\hline Metropolitana & 21.5 & 31.1 & 18.2 & 4.1 & 2.5 & 2.3 & 1.5 & 18.7 \\
\hline Paracentral & 13.0 & 38.5 & 14.2 & 1.2 & 3.0 & 1.8 & 2.4 & 26.0 \\
\hline Oriental & 17.9 & 35.4 & 9.9 & 4.7 & 4.0 & 6.2 & 2.2 & 19.7 \\
\hline \multicolumn{9}{|l|}{ Estrato } \\
\hline Alto & 22.9 & 45.8 & 3.6 & 7.2 & 2.4 & 1.2 & 4.8 & 12.0 \\
\hline Medio-alto & 14.7 & 50.6 & 12.9 & 7.1 & 1.8 & 1.2 & 1.2 & 10.6 \\
\hline Medio-bajo & 22.0 & 33.9 & 22.0 & 3.0 & 2.4 & 1.2 & 0.0 & 15.5 \\
\hline Obrero & 18.0 & 29.6 & 15.6 & 3.2 & 1.6 & 2.4 & 2.4 & 27.2 \\
\hline Marginal & 17.4 & 20.5 & 15.5 & 2.5 & 6.8 & 5.6 & 4.3 & 27.3 \\
\hline Rural & 14.3 & 32.2 & 11.1 & 1.5 & 2.0 & 6.9 & 0.7 & 31.4 \\
\hline \multicolumn{9}{|l|}{ Sexo } \\
\hline Masculino & 17.7 & 30.5 & 17.7 & 3.7 & 3.5 & 3.0 & 2.0 & 21.8 \\
\hline Femenino & 16.6 & 36.8 & 10.2 & 2.9 & 1.7 & 4.6 & 1.5 & 25.5 \\
\hline \multicolumn{9}{|l|}{ Edad } \\
\hline 18 a 25 años & 17.3 & 40.2 & 12.3 & 2.9 & 2.3 & 0.9 & 1.8 & 22.3 \\
\hline 26 a 40 años & 16.9 & 31.7 & 13.5 & 3.0 & 3.2 & 4.6 & 1.6 & 25.4 \\
\hline 41 a 55 años & 17.0 & 29.5 & 15.4 & 4.6 & 2.9 & 5.4 & 2.9 & 22.4 \\
\hline 56 años y más & 17.4 & 33.5 & 15.5 & 3.1 & 0.6 & 5.6 & 0.6 & 23.6 \\
\hline \multicolumn{9}{|l|}{ Estudios } \\
\hline Ninguno & 18.3 & 29.4 & 5.9 & 2.0 & 2.0 & 10.5 & 1.3 & 30.7 \\
\hline Primaria & 15.1 & 29.9 & 12.1 & 2.5 & 2.7 & 5.5 & 1.6 & 30.5 \\
\hline Plan básico & 15.5 & 34.0 & 13.4 & 1.0 & 2.1 & 3.1 & 2.1 & 28.9 \\
\hline Bachillerato & 16.8 & 36.8 & 16.8 & 3.6 & 3.6 & 0.8 & 1.6 & 20.0 \\
\hline Superior & 20.5 & 38.5 & 18.0 & 6.5 & 2.2 & 1.4 & 2.2 & 10.8 \\
\hline \multicolumn{9}{|l|}{ Religión } \\
\hline Ninguna & 22.3 & 27.2 & 18.0 & 2.9 & 3.4 & 3.9 & 1.5 & 20.9 \\
\hline Católica & 16.3 & 36.1 & 13.6 & 3.6 & 1.8 & 2.9 & 2.0 & 23.7 \\
\hline Evangélica & 12.9 & 32.9 & 10.8 & 3.2 & 4.0 & 7.2 & 1.6 & 27.3 \\
\hline Otra & 39.1 & 26.1 & 17.4 & 0.0 & 4.3 & 0.0 & 0.0 & 13.0 \\
\hline
\end{tabular}

Si las elecciones fueran el próximo domingo, ¿por cuál partido votaría Ud.? 
dría haccr algo" (27.5 por ciento). En el caso del Centro Democrático Unido, la mayor parte de las respuestas apuntaban al candidato como la razón para prelerir al partido (65.9 por ciento); mientras que cl caso del PDC se destacaron las razones de que "podría hacer algo" (25 por ciento), por tradición política (21.9 por ciento) y por "simpatía" (18.8 por ciento).

\section{Conclusiones}

La encuesta permite concluir varios aspectos sobre la opinión pública a finales de 1998.

- Los principales problemas del país siguen siendo los mismos a finales de 1998: delincuencia y economía. Sin embargo, los salvadoreños no eslán preocupados con la misma magnitud que en el pasado por cierto aspecto de la economía nacional, esto es, la inflación.

- La mayoría de los ciudadanos cree que la pobreza aumentó en el año que finaliza. Esta convicción está también a la base del pensamiento de quc la siluación económica nacional y familiar se mantiene igual.

- La impresión, no obstante, de que la economía ha empeorado durante 1998 no ha sido del mismo nivel que en años anteriores. En esta ocasión, la mayoría de gente se decantó en decir que la economía se mantiene igual, a diferencia del pasado cuando la opinión más frecuente era de que había cmpeorado.

- Dos de cada tres salvadoreños opinaron de que el nivel de criminalidad en el país aumentó durante 1998. Pero, el porcentaje de personas que lucron víctimas de algún hecho delincuencial fue más bajo que el obtenido en años anteriores. Ello sugiere que la preocupación por el crimen ha rolo cl anclaje con la realidad.

- A nivel de evaluación del gobierno, la administración Calderón Sol continúa obteniendo los mismos logros (infraestructura y educación) y fracasos (delincuencia y economía) que le han caracterizado en la opinión pública. Sin embargo, en términos de valoración general, el gobierno alcanzó una imagen que, sin llegar a ser positiva, se acerca a su mejor situación frente a la opinión pública.

- En términos electorales, los salvadoreños siguen mostrando elevados niveles de desconfianza y desinterés en el proceso eleccionario. Estas actitudes son especialmente fuertes entre los ciudada- nos que no tienen partido político o que no han decidido un partido político.

- La simpatías por los candidatos presidenciales que están compitiendo por las elecciones del 99 se distribuyen y favorecen al candidato Francisco Flores de ARENA. La encuesta muestra un virtual empate en términos de imagen personal entre los postulantes Guardado del FMLN y Zamora del CDU. El resto de candidatos enfrentan problemas de reconocimiento entre la población, es decir, el porcentaje de salvadoreños que los conocen o han oído de ellos es muy bajo.

- En términos de intención de voto por partido, el partido de gobierno encabeza las preferencias - para mediados de diciembre-, le sigue a gran distancia el FMLN que ocupa el segundo lugar. Luego, con otra diferencia notable, el tercer lugar lo tiene aparentemente el CDU, pero a corta distancia del PDC y del resto de partidos pequeños.

- La encuesta reveló que la imagen de los candidatos pesa en la elección del partido. El candidato Flores, por ejemplo, recoge muchas más simpatías que su partido, con el candidato del FMLN pasa lo opuesto y en el caso de Zamora, éste obtiene, por mucho, más simpatía que su partido, el cual es muy poco conocido.

Buena parte de los datos presentados en este artículo y que fueron obtenidos en la encuesta de diciembre del IUDOP, ofrecen indicios para explicar algunos estados de la opinión a finales de 1998, pero sobre todo brindan pistas para entender lo que puede ser la conducta electoral de los ciudadanos en el primer trimestre del año 99.

\section{Referencias bibliográficas}

Cruz, José Miguel, Elecciones y pensamiento social: opinión pública en los comicios 97. San Salvador (Mimeo), 1997.

Instituto Universitario de Opinión Pública (IUDOP), Encuesta de evaluación del año 1997, Serie de informes 66, San Salvador: IUDOP-UCA, 1998a.

Instituto Universitario de Opinión Pública (IUDOP), Encuesta de opinión sobre delincuencia, Serie de informes 70, San Salvador: IUDOP-UCA, $1998 b$.

Instituto Universitario de Opinión Pública (IUDOP), Encuesta de evaluación del año 1998, Serie de informes 72, San Salvador: IUDOP-UCA, 1999.

Instituto Universitario de Opinión Pública (IUDOP), "Los salvadoreños evalúan la situación del país a finales de 1996", Encuesta de opinión pública, Estudios Centroamericanos (ECA), 1997. 579-580, pp. 55-68. 